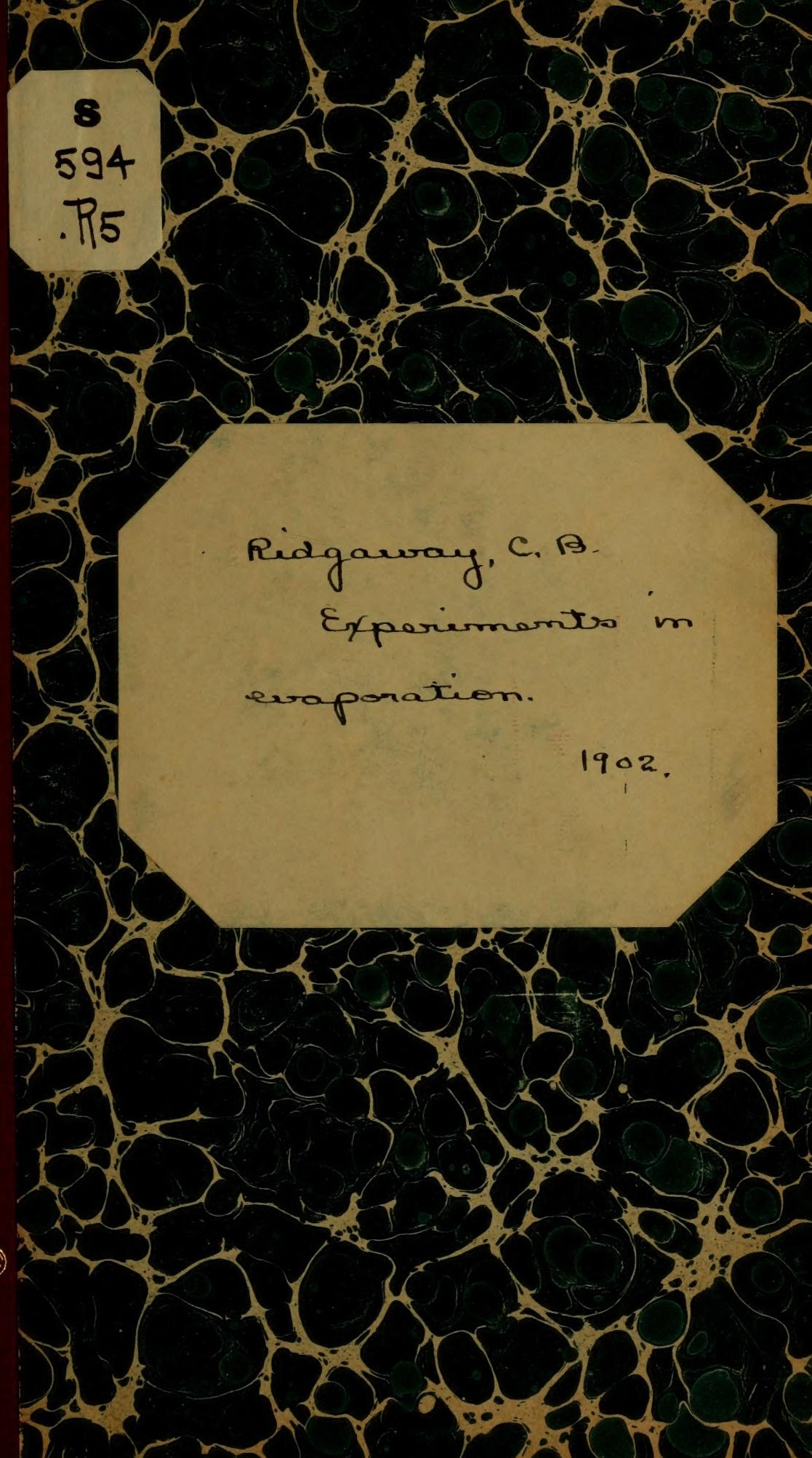




$$
\text { ช }
$$




\title{
UNIVERSITY OF WYOMING.
}

Agricultural College Department.

\section{WYOMING EXPERIMENT STATION,}

LARAMIE, WYOMING.

\section{BULIETIN NO. 52. \\ APRIL, 1902.}

\section{Experiments in Evaporation.}

BY C. B. RIDGAWAY.

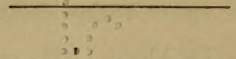

Bulletins will be sent free upon request. Address : Directur Experiment Station, Laramie, Wyo.
\end{abstract}

Monograph

IIBRARY OF LUNGRESS, RECEIVED

AUG 211902

BMEIDN OF DOCUMENTS. 


\section{Wyoming Agricultungl Experiment Station.}

\section{UNIVERSITY OF WYOMING.}

\section{BOARD OF TRUSTEES.}

Hon. OTTO GRAMM, President, Laramie................1903

Hon. GRACE RAYMOND HEBARD, B.S.,Ph.D.,Sec'y, Cheyenne.1903 Hon. HENRY L. STEVENS, M. D., Laramie................1903 Hon. TIMOTHY F. BURKE, LL. B., Vice President, Cheyenne..1907 Hon. JOHN C. DAVIS, Treasurer, Rawlins............... 1907 Hon. MORTIMER JESURUN, M. D., Douglas.............. 1907 Hon. ARTHUR C. JONES, Laramie...................... 1903 Hon. JOHN A. BECKWITH, Evanston.................. 1905 Hon. S. CONANT PARKS, Ph. D., Lander.................. 1905 State Supt. of Public Instruction T. T. TYNAN .......... Ex-officio President ELMER E. SMILEY, A. M., D. D............ Ex-officio

\section{Agricultural Committee of the Board of Trustees.}

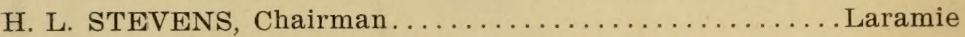

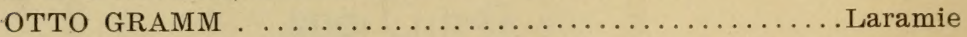
A. C. JONES Laramie

President of the University of Wyoming.

ELMER E. SMILEY, A. M., D. D.

STATION COUNCIL.

E. E. SMiley, A. M., D. D.. $2 \ldots \ldots \ldots \ldots \ldots \ldots$. F. E. EMERY, M. S., Vice Director, Agriculturist and Horticulturist

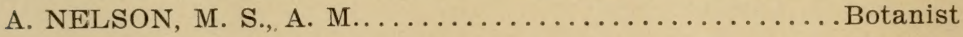

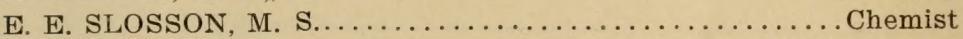

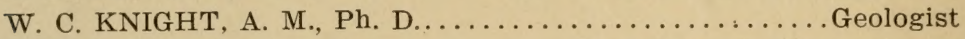
C. B. RIDGAWAY, A. M............. Physicist and Meteorologist

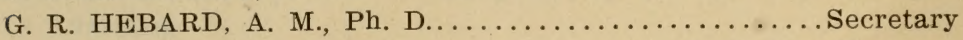
BURTON P. FLEMING, B. S............... Irrigation Engineer ELIAS E. NELSON, A. M............... Assistant Horticulturist E. E. SIGMAN ................... Foreman Experiment Farm 


\section{IN'TRODUCTION.}

The two great questions which are of most importance to the agriculturist in this section of the country are: How may we best conserve and utilize the available water to the greatest advantage? and, How may we eliminate the surplus alkali from the soil?

It was in the hope of throwing some light upon the solution of these great questions that the following experiments were undertaken.

I regret to state that the young man who took the observations for a few days, on account of the necessary absence of the regular observer, lost the records for two months of the year I900. I have concluded, therefore, to use the records for I9OI only in reporting the amount of evaporation. The results in regard to the rise of alkali, however, will cover the period of two years.

The water used in these experiments was obtained from the artesian well near the University, and the amount of alkali contained therein was .639 grains per gallon as per analysis made by Professor E. E. Slosson, Chemist of the Station.*

I wish here to acknowledge my indebtedness to Mr. B. P. Fleming, Irrigation Engineer, for all of the data found in Table II.

*Bulletin No. 24, Wyo. Ag. Exp. Sta. Water Aralyses. 


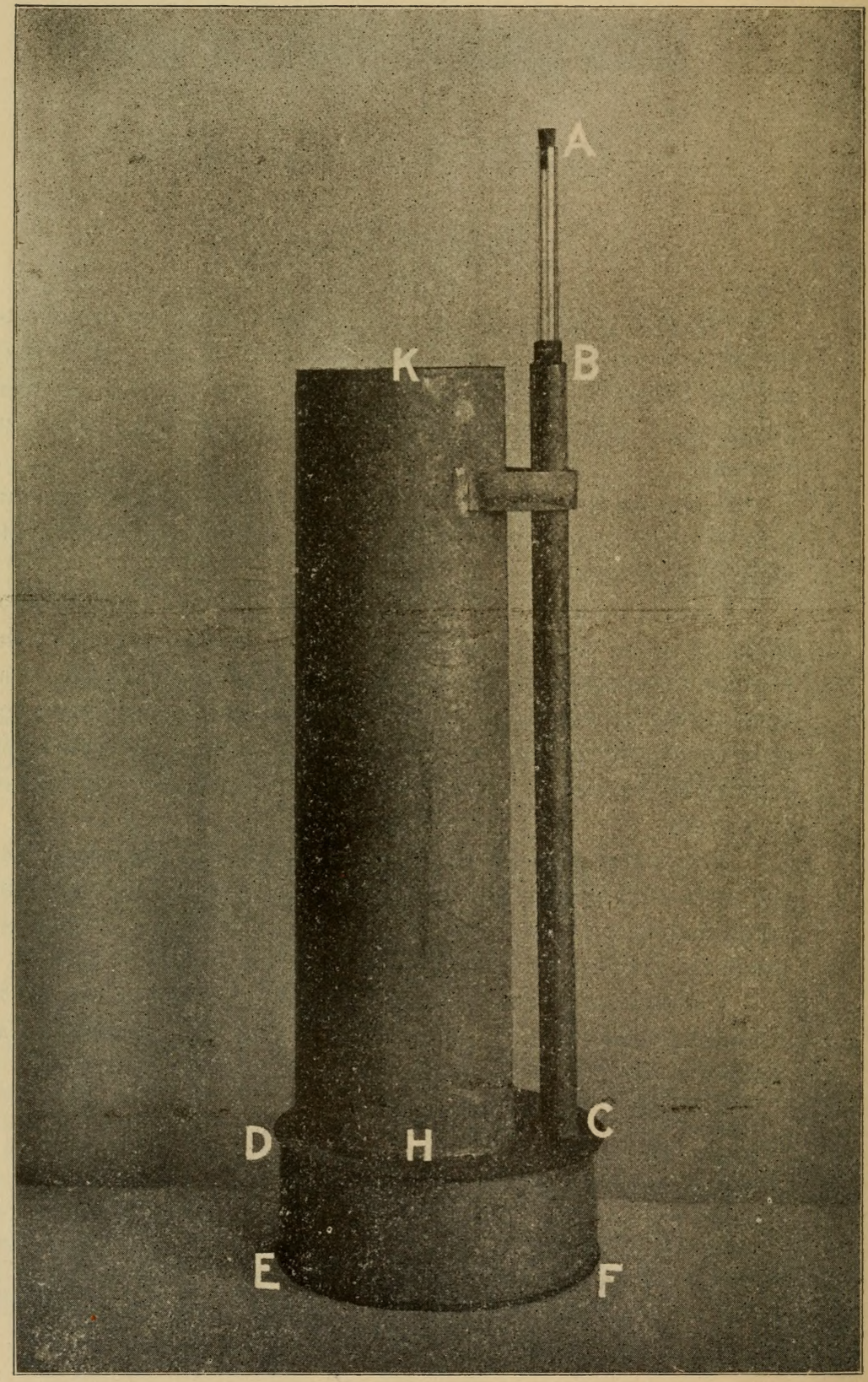




\section{Experiments in Evaporation.}

The following were some of the questions which I hoped to partially answer by these experiments:

I. How rapidly does moisture evaporate from the soil when the level of water is kept at a certain distance from the surface by subirrigation?

2. What effect has stirring the soil once a week to certain depths upon the evaporation of moisture when the level of water is maintained at a constant depth?

3. What effect has subirrigation upon the rise of alkali?

4. What effect has alkali upon the evaporation of moisture when the level of water is kept at a certain depth from the surface?

For the purpose of investigating these questions I had constructed 15 pieces of apparatus similar to the one shown in the cut. D E F C is a drum, 9 inches in diameter and 4 inches deep, made of heavy galvanized sheet iron. $\mathrm{K} \mathrm{H}$ is a tube of the same material, 26 inches long and 6 inches in diameter, extending to the bottom of the drum, and with its lower surface perforated with many holes so as to admit water freely from the drum.

This pipe was filled with soil in place on the experiment farm by driving the tube into the soil as far as possible, then with trowels and knives cutting the soil from around the tube and driving it down still further. The process was continued until the tube was entirely filled. The tube was then soldered into the drum around the rim at $\mathrm{H}$.

The tube B C is of galvalized iron pipe soldered into the drum at $\mathrm{C}$ and fastened to the larger pipe by a support a little below $\mathrm{B}$. 
This apparatus was buried in the ground on the experiment plat so that the top of the tube $\mathrm{H} \mathrm{K}$ was level with the surface of the soil. Thus I had the undisturbed soil from the farm buried in the ground with the top exposed to the wind, rain, and sunshine, and having the same temperature as the surrounding earth.

The tank, from which observations were taken of the amount of evaporation of moisture from the water surface, and the soil thermometers, for determining the temperature of the soil at various depths, were located about 16 feet trom the buried tubes.

In the tube $\mathrm{B}$ C was put a small empty vial fitted with a rubber stopper. In the stopper was inserted an aluminum wire long enough to reach up to a certain mark on the glass tube $\mathrm{B}$ A when the vial was floating on the water kept at a certain level in the tube B C. The tube B A was calibrated so that 5.5 grams of water poured into the tube B C would cause the end of the wire to rise through one space. The upper end of the wire was bent at a right angle, and the lower end of the tube at $B$ was almost closed by fusion, so that the pointer on the end of the wire could move up or down through the length of the tube but could not fall below the lower end $\mathrm{B}$.

The level of water in the tube B C was kept at about the same height by pouring water from a graduate into the tube $\mathrm{A} \mathrm{B}$ at 7 p. m. each day during the crop season of I9oo. During the year I9or, the method of obtaining the amount of water used each day differed somewhat from the method used the previous year. A beaker nearly full of water was first carefully weighed, then enough water poured from it into the tube $\mathrm{B} \mathrm{C}$ to bring the floating index up to the required mark; the beaker was again weighed and the amount in grams of water poured into the tube was ascertained and recorded. Very frequently the index would come above the proper mark, then an allowance had to be made for the excess poured in; an allowance also had to be made for the amount of evaporation the 
next day. This, however, was very easily accomplished by means of the calibrated tube A B.

DATA IN REGARD TO THE DIFFERENT TUBES.

No. I was kept full of water to within 6 inches of the top. No. 2 was kept full of water to within 12 inches of the top.

No. 3 was kept full of water to within 18 inches of the top.

No. 4 was kept full of water to within 22 inches of the top.

No. 5 was kept full of water to within 22 inches of the top and the surface stirred once a week to the depth of 2 inches.

No. 6 was kept full of water to within 22 inches of the top and the surface stirred once a week to the depth of 4 inches.

No. 7 was kept full of water to within 22 inches of the top and the surface stirred once a week to the depth of 6 inches.

In tubes $8,9,10$, and I I enough sodium carbonate in solution ( 5 per cent) was poured in A C to moisten each tube full of earth and leave the solution standing at the proper level, after which artesian well water was added each day. In tubes I2, I3, I4, and I5 a Io per cent solution of sodium carbonate was added till all of the earth was moistened with the liquid, then water was poured in as in the other tubes.

No. 8 was kept full of water to within 6 inches of the top.

No. 9 was kept full of water to within 12 inches of the top.

No. Io was kept full of water to within 18 inches of the top.

No. I I was kept full of water to within 22 inches of the top.

No. I2 was kept full of water to within 6 inches of the top.

No. I3 was kept full of water to within 12 inches of the top.

No. I4 was kept full of vater to within 18 inches of the top.

No. I 5 was kept full of water to within 22 inches of the top.

In column I6, of Table No. I, the amount of evaporation from the free water surface will be found, while column $I 7$ of the same table contains the amount of rainfall.

The amounts in Table No. I are recorded in linear inches, while those in Table No. II represent the per cent of alkali found in the soil at various depths. 
The following conclusions were drawn from these experiments:

The evalyoration from the surface of the soil with the level of water maintained at 6 inches below was 95 per cent, at I 3 inches below it was 70 per cent, at 18 inches below it was 45 per cent, and at 22 inches below it was 35 per cent of what it was at the surface of the water in the evaporation tank.

Stirring the ground once a week to the depth of 2 inches retarded evaporation to the amount of 19 per cent, when stirred to a depth of 4 inches it was retarded 23 per cent, and when stirred to a depth of 6 inches evaporation was retarded 45 per cent. The water in all three of the pipes was maintained at a depth of 22 inches below the surface of the soil.

Evaporation was retarded 43 per cent when the soil contained .0597 per cent of alkali and the level of water was maintained at 6 inches below the surface. The amount of retardation was 55 per cent in soil containing .5116 per cent of alkali, and the level of water 12 inches below the surface. In soil containing .5375 per cent of alkali the amount of retardation was 50 per cent when the level of water was maintained at 18 inches below the surface; while the retardation was 57 per cent in soil containing .6205 per cent of alkali and the level of water kept at 22 inches below the surface. The amount of alkali is the average amount found in the entire 26 inches of soil.

More alkali was found in the first three inches than in any other three inches of the soil.

More alkali was found in the last two inches than in the three next above.

The following analysis of the soil used in these experiments was made by Professor E. E. Slosson in 1892, and published in Bulletin No. 6 . 
Chemical Analysis of the Soil of the Laramie Experiment Farm.

Analysis of fine earth.
Insoluble matter . . . . . .

Surface Subsoil

1-9 in. $\quad 9-18$ in.

Soluble silica.

$80.11 \quad 77.28$

Potash $\left(\mathrm{K}_{2} \mathrm{O}\right)$.

2.78

1.92

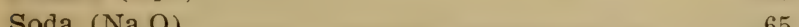

.53

Lime $(\mathrm{CaO})$

$.65 \quad .45$

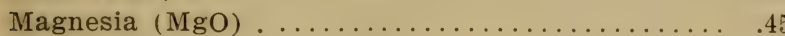

3.77

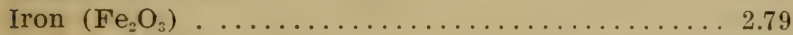

.08

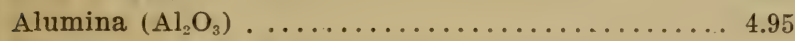

2.49

Phosphoric acid $\left(\mathrm{P}_{2} \mathrm{O}_{5}\right) \ldots \ldots \ldots \ldots \ldots \ldots \ldots \ldots \ldots \ldots . .14 \quad .15$

4.71

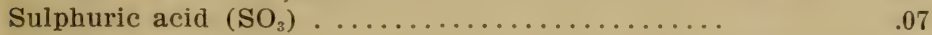

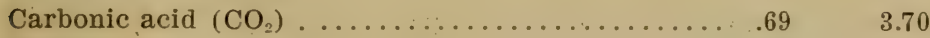

Moisture...................... $1.87 \quad 1.24$

Volatile and combustible matter ............... 312

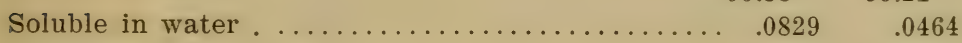

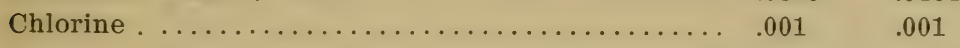


Wyoming Experiment Station.

\begin{tabular}{|c|c|c|c|}
\hline$=$ & ๕. 읍 ㅇํำ & 勇 & \\
\hline$\cong$ & 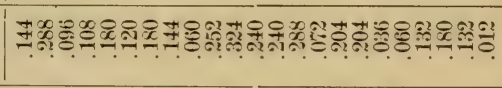 & \begin{tabular}{|l} 
题 \\
is
\end{tabular} & 은 \\
\hline$\stackrel{2}{2}$ & 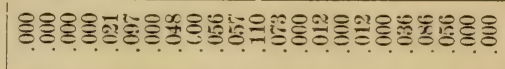 & 兽 & tas. \\
\hline \pm & 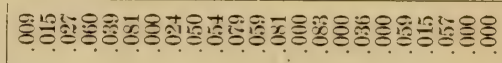 & $\frac{0}{\alpha}$ & 范 \\
\hline 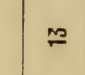 & 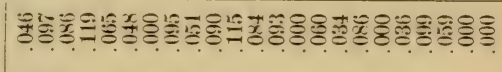 & 絪 & 谙 \\
\hline$\simeq$ & 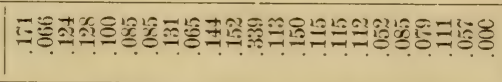 & $\left|\begin{array}{c}6 \\
6 \\
b \\
a \\
0\end{array}\right|$ & $\stackrel{\leftrightarrow}{\circ}$ \\
\hline$=$ & 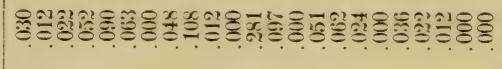 & $\mid \begin{array}{c}0 \\
\stackrel{x}{0} \\
\end{array}$ & 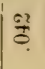 \\
\hline 으 & 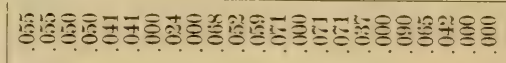 & $\overrightarrow{\widetilde{\xi}}$ & 彩 \\
\hline$\sigma$ & 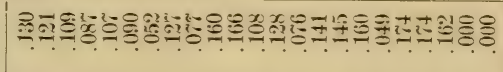 & $\left|\begin{array}{c}1 \\
0 \\
0 \\
0 \\
0\end{array}\right|$ & 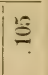 \\
\hline$\infty$ & | & $\left|\begin{array}{c}\pi \\
= \\
-\pi\end{array}\right|$ & ?ִ. \\
\hline$n$ & 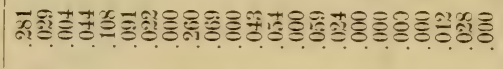 & 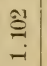 & 율. \\
\hline$\infty$ & 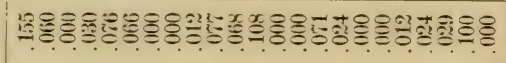 & $=1$ & 菅 \\
\hline is & 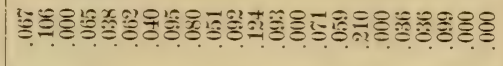 & $\mid$ & 总 \\
\hline+ & 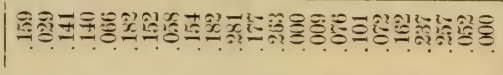 & 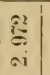 & T! \\
\hline m & 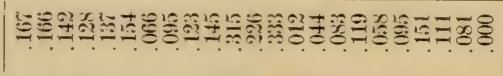 & 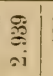 & $\frac{\hat{g} \mathfrak{g}}{2}$ \\
\hline$\sim$ & 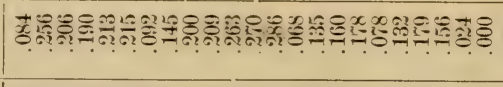 & $\left|\begin{array}{l}\overrightarrow{2} \\
2 \\
\infty \\
\infty\end{array}\right|$ & is \\
\hline- & 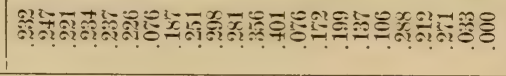 & 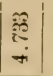 & $\stackrel{\square}{\Xi}$ \\
\hline & $\operatorname{\theta ig}=2 \Re$ & : & \\
\hline
\end{tabular}




\begin{tabular}{|c|c|c|c|c|}
\hline$=$ & $\stackrel{9}{\stackrel{\circ}{ }}$ & & 蛋: & \\
\hline 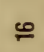 & 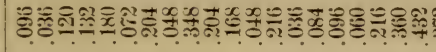 & 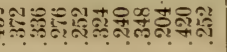 & & \& \\
\hline 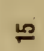 & 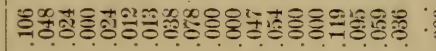 & 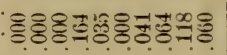 & & Fे \\
\hline \pm & 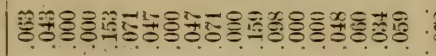 & 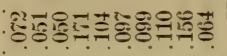 & & 荌 \\
\hline 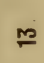 & 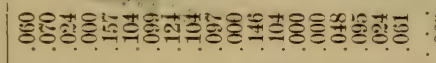 & 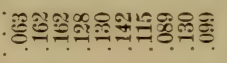 & & tg \\
\hline$\cong$ & 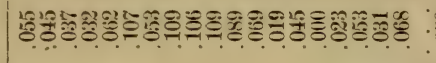 & 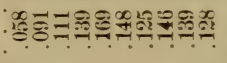 & 䜌 & 褋 \\
\hline$=$ & 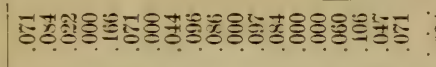 & 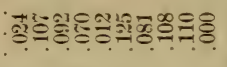 & $\mid$ & $\overline{\mathrm{g}}$ \\
\hline 둔 & 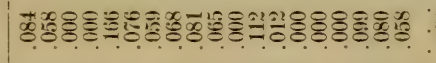 & 12. & $\left|\begin{array}{ll}\infty \\
a \\
\vdots \\
\hdashline\end{array}\right|$ & 营 \\
\hline os & 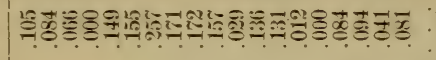 & 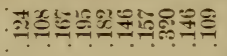 & 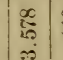 & $\fallingdotseq$ \\
\hline$\infty$ & 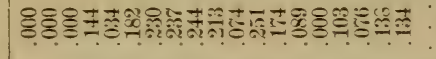 & 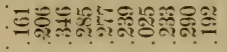 & 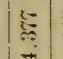 & 19 \\
\hline n & 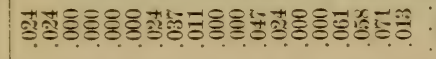 & 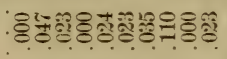 & : & 管 \\
\hline - & 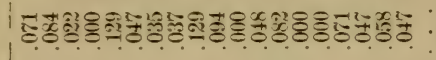 & 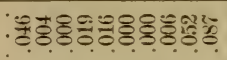 & $\overrightarrow{\tilde{\xi}}$ & I \\
\hline م & 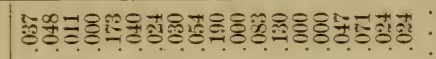 & 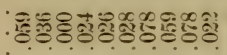 & $\mid$ & 导 \\
\hline+ & 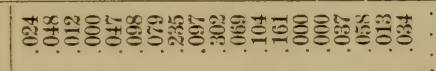 & 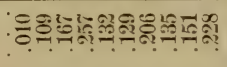 & $\frac{9}{2}$ & $\stackrel{\circ}{\xi}$ \\
\hline$m$ & 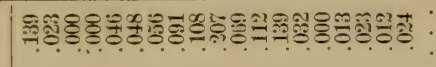 & 武석 & ig & $\stackrel{\leftrightarrow}{\stackrel{s}{\circ}}$ \\
\hline a & 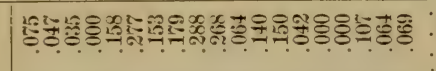 & 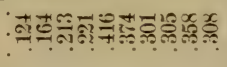 & $\mid$ & $\frac{\mathrm{g}}{6}$ \\
\hline - & | & 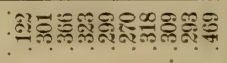 & 귘 & $\frac{1}{9}$ \\
\hline
\end{tabular}




\begin{tabular}{|c|c|c|c|c|}
\hline & & 高 & 翅 & \\
\hline & & 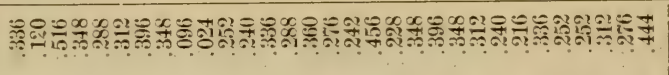 & 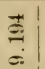 & 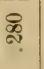 \\
\hline & & 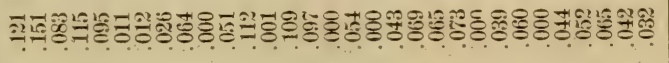 & 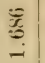 & 荌 \\
\hline & t & 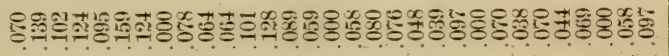 & 紊 & 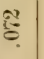 \\
\hline & $=$ & 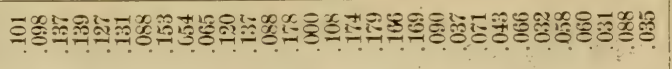 & 管 & 管 \\
\hline & 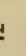 & 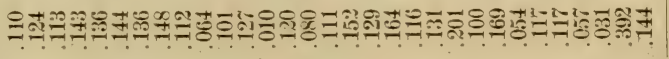 & : & 魚 \\
\hline & $=$ & 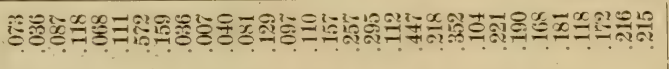 & $\stackrel{3}{2}=$ & 象 \\
\hline & 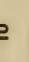 & 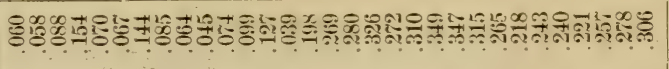 & $\frac{\infty}{z}$ & $\underset{7}{q}$ \\
\hline & s & 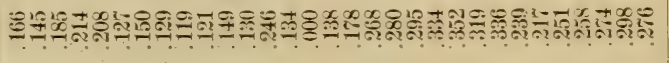 & 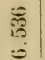 & है? \\
\hline & 。 & 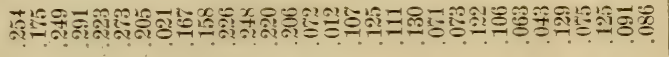 & 集 & 竺 \\
\hline & - & 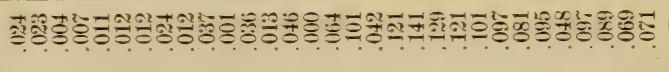 & 产i & 8 \\
\hline & 。 & 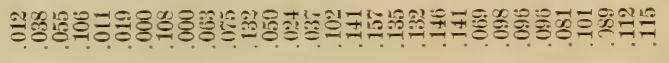 & 苦 & के \\
\hline & م & 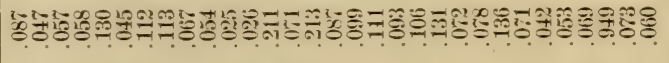 & $\begin{array}{l}0 \\
0 \\
6 \\
0 \\
0\end{array}$ & $\stackrel{ \pm}{*}$ \\
\hline & + & 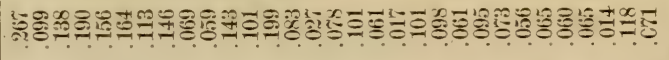 & 8 & 彦 \\
\hline & b & 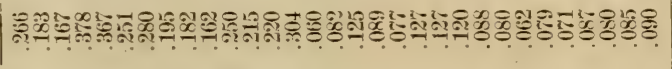 & $\underset{d}{d}$ & 总 \\
\hline & $\sim$ & 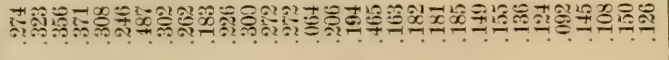 & 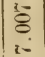 & ๙ั? \\
\hline$E$ & - & 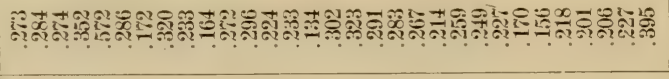 & 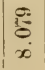 & : \\
\hline 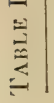 & & & 亥 & $\frac{\bar{g}}{\Sigma}$ \\
\hline
\end{tabular}




\begin{tabular}{|c|c|c|c|}
\hline$\overline{z_{i}} \mid \bar{a}$ & & 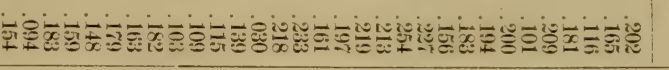 & - \\
\hline$\overline{\mathrm{B}}$ & & 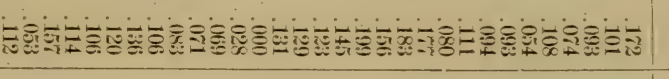 & $\sim$ \\
\hline 总 & 8 & 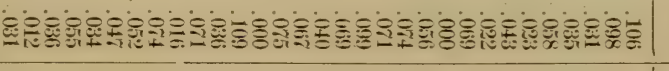 & w \\
\hline 辈 & & 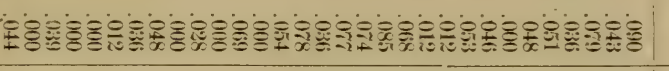 & + \\
\hline 昰 & 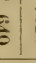 & 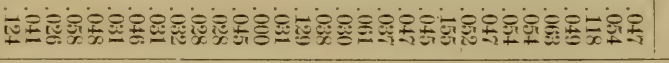 & on \\
\hline 禹施 & 童 & 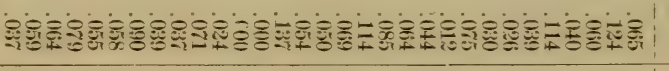 & $\infty$ \\
\hline$\underbrace{2}$ & & 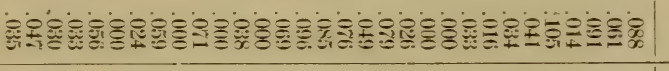 & $\sim$ \\
\hline$\underbrace{2}_{i=1}$ & & 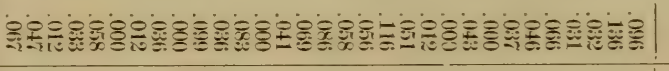 & $\infty$ \\
\hline$-\frac{1}{x} \mid$ & & 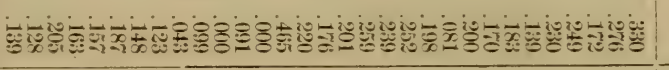 & $\infty$ \\
\hline 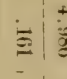 & & 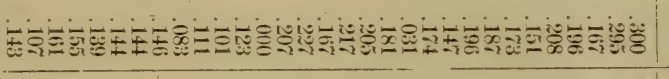 & a \\
\hline 5 & & 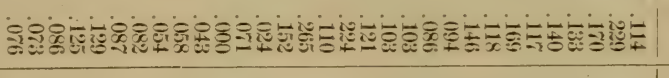 & $=$ \\
\hline 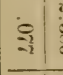 & & 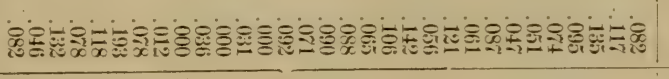 & $\vec{N}$ \\
\hline 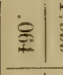 & & 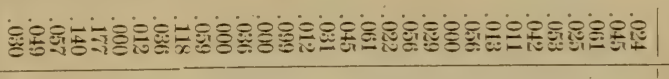 & | \\
\hline 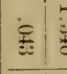 & & 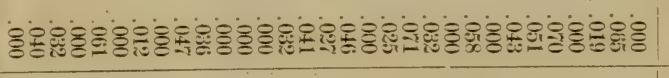 & $\vec{f}$ \\
\hline 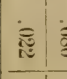 & & 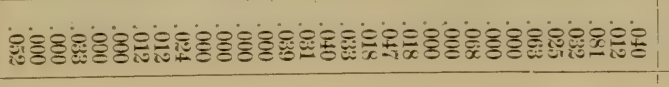 & जे \\
\hline$\left|\vec{\alpha}_{-2}\right|$ & & 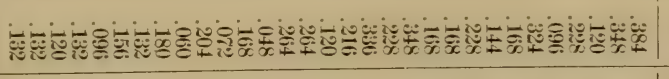 & क \\
\hline & & 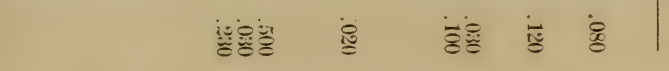 & $=$ \\
\hline
\end{tabular}




\begin{tabular}{|c|c|c|c|c|c|}
\hline & & 8 & है. & & \\
\hline & 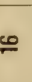 & 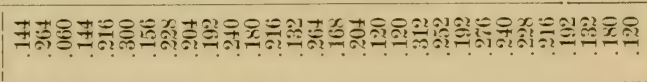 & 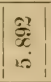 & 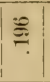 & ज़ \\
\hline & مِ & 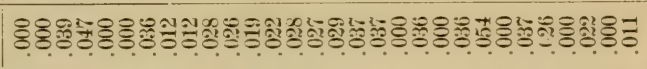 & 药 & Fi & 筒 \\
\hline & \pm & 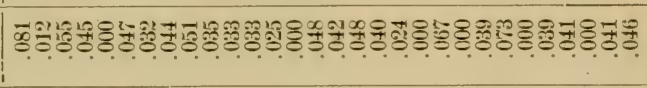 & 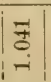 & 争 & 쿵. \\
\hline & 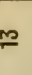 & 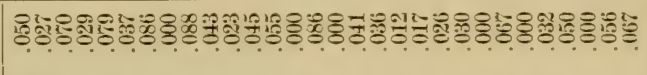 & $\mid$ & $\mid \infty$ & gु \\
\hline & $\simeq$ & 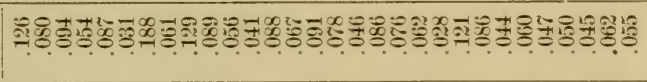 & $\left|\begin{array}{cc}R \\
0 \\
\therefore i\end{array}\right|$ & 产 & $\stackrel{8}{9}$ \\
\hline & $=$ & 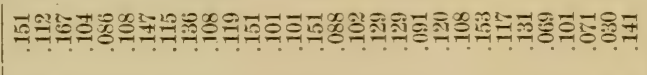 & 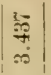 & $=12$ & g: \\
\hline & 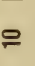 & 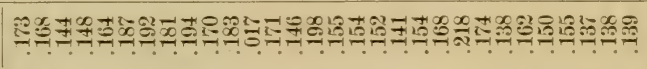 & $\mid$ & $\stackrel{2}{\rightarrow 2}$ & $\overrightarrow{9}$ \\
\hline & or & 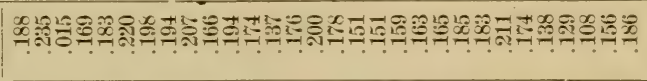 & 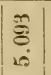 & : & $\stackrel{0}{?}$ \\
\hline & $\infty$ & 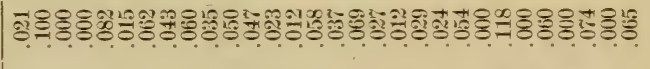 & $\mid$ & 鄯 & $\because$ \\
\hline & r & 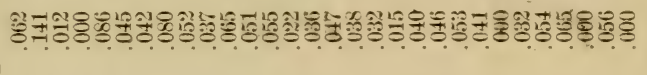 & 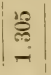 & 滓 & 핳. \\
\hline & ه & 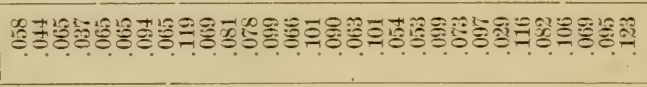 & 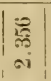 & 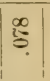 & 实 \\
\hline & مו & 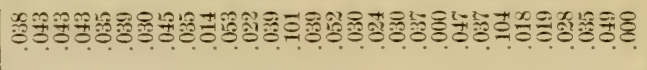 & $\mid$ & $\mid \frac{\infty}{0}$ & 象. \\
\hline & $\sigma$ & 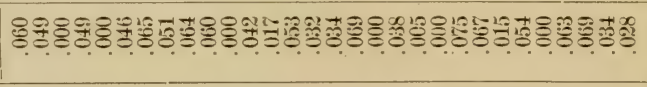 & $\mid \begin{array}{c}\text { 嵒 } \\
-1\end{array}$ & $\mid$ & iz \\
\hline & $\mathrm{m}$ & 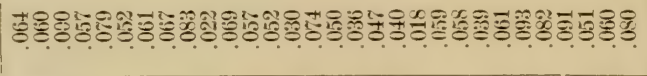 & 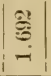 & 容 & 总 \\
\hline & $\sim$ & 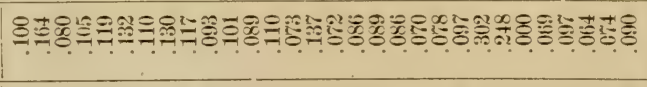 & $\left|\begin{array}{l}\infty \\
0 \\
\infty \\
\infty\end{array}\right|$ & $|\ddot{\leftrightarrow}|$ & i \\
\hline & - & 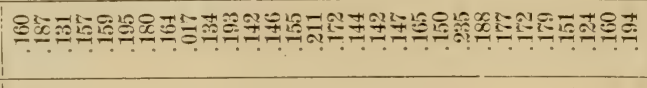 & 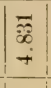 & $\vec{\Xi}$ & 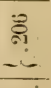 \\
\hline & & 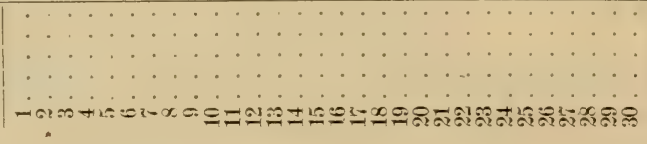 & $|\dot{\Xi}|$ & $\left|\begin{array}{c}\vdots \\
\vdots \\
\tilde{\Xi} \\
\frac{\tilde{d}}{2}\end{array}\right|$ & 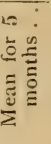 \\
\hline
\end{tabular}


TABLE II-Per cent of Alkali.

\begin{tabular}{|c|c|c|c|c|c|c|c|c|}
\hline Depth in inches. & 8 & 9 & 10 & 11 & 12 & 13 & 14 & 15 \\
\hline $3 \ldots \ldots$ & .1232 & .4972 & .6144 & 1.48 & .2076 & 1.7916 & .9724 & 1.2492 \\
\hline $6 \ldots \ldots$ & .1044 & .2176 & .2728 & .5560 & .1924 & .7048 & .6104 & .6708 \\
\hline $9 \ldots \ldots$ & .0360 & .1348 & .1552 & .3000 & .1928 & .4048 & .5824 & .5904 \\
\hline $12 \ldots \ldots$ & .0480 & .1516 & .2048 & .2316 & .2244 & .2544 & .5596 & .5752 \\
\hline $15 \ldots \ldots$. & .0472 & .0912 & .2660 & .1360 & .2236 & .2468 & .3784 & $.50 \subset 0$ \\
\hline $18 \ldots \ldots$ & .0404 & .0380 & .2064 & .1688 & .2648 & .2168 & .4180 & .5140 \\
\hline $22 \ldots \ldots \ldots$ & .0376 & .0316 & .2064 & .3224 & .2616 & .2344 & .2924 & .4024 \\
\hline $25 \ldots \ldots$ & .0412 & .0360 & .1796 & .3256 & .1400 & .2392 & .4904 & .4620 \\
\hline Sum ........ & .4780 & 1.1980 & 4.3040 & 4.9640 & 1. 7072 & 4.0928 & 2.1156 & 3.5205 \\
\hline 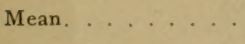 & .0597 & .1497 & .2644 & $: 4401$ & .2134 & .5116 & .5375 & .6205 \\
\hline
\end{tabular}




\section{BLLLETINS OF THE WYOMING AGRICULTURAL EXPERIMENT STATION}

\section{These bulletins are sent free of charge to any address upon ap- plication to the Director of Experiment Station, Laramie, Wyo.}

(Only the bulletins named below are available for distribution.)

No. 7-July, 1892 . Insecticides.

No. 8-October, 1892. Irrigation and Duty of Water.

No. 9-December, 1892. Sugar Beets in Wyoming in 1892.

No. 11-February, 1893. Crop reports for 1892.

No. 12-April, 1893. Ground Squirrels (Gophers).

No. 13-July, 1893. The Feeding and Management of Cattle.

No. 14-October, 1893. Geology of the Wyoming Experiment Farms, and Notes on the Mineral Resources of the State.

No. 15-December, 1893. The Winter-Killing of Trees and Shrubs.

No. 16-December, 1893 . Grasses and Forage Plants.

No. 17-March, 1894. I. Crop Report for 1893. II. Cost and Profit of Growing Wheat. III. Sugar Beets. IV. Garden Vegetables and Tobacco. V. Meteorology for 1893

No. 19-September, 1894. Squirrel Tail Grass (Fox-Tail).

No. 20-October, 1894. The Artesian Wells of Southern Wyoming.

No. 21.-January, 1895. The Grain Smuts and Potato Scab.

No. 22-April, 1895. I. Onions. II. Crop Reports, 1894. 1, Potatoes; 2, Turnips and Other Root Crops; 3, Grasses and Forage Plants; 4, Cereals; 5, Other Crops. III. Cost and Profit of Growing Wheat. IV. Small Fruits at Laramie.

No. 23-May, 1895. Notes on Climate.

No. 24-August, 1895. Water Analyses.

No. 25-November, 1895. Results of Three Years' Experiments in Cost and Profit of Growing Wheat.

No. 26-December, 1895 . Garden Peas.

No. 27-March, 1896. Meteorology for 1895, and Notes on Climate from 1891 to 1896.

No. 28-May, 1896. First Report on the Flora of Wyoming.

No. 29-July, 1896. Alkali.

No. 30-September, 1896. Stock Feeding Experiments at Lander.

No. 31-December, 1896. The Worst Weeds of Wyoming and Suggested Weed Legislation.

No. 32-March, 1897. Potatoeș.

No. 33-June, 1897. The Composition of Prepared Cereal Foods.

No. 34-November, 1897. Fruit Growing in Wyoming.

No. 35-December, 1897. Mechanical Analyses and Water Content of Wyoming Soils.

No. 36-April, 1898. Wyoming Sugar Beets.

No. 37-June, 1898. Stooling of Grains.

No. 38-September, 1898. Cultivated Shade and Forest Trees.

No. 39-December, 1898. Alkali Studies, II.

No. 41-November, 1899. Some Experiments With Subsoiling.

No. 42-December, 1899. Some Native Forage Plants for Alkali Soils.

No. 43-March, 1900. Alfalfa as a Hay Crop.

No. 44-April, 1900 . Alfalfa as a Fertilizer.

No. 45-June, 1900. Artesian Basins of Wyoming.

No. 46-January, 1901. The Brome-Grasses of Wyoming.

No. 47-April, 1901. Lamb Feeding Experiment.

No. 48-May, 1901. Experiments in Wheat Culture.

No. 49-June, 1901. Alkali Lakes and Deposits.

No. 50-March, 1902. Native Vines in Wyoming Homes.
No. 51-May, 1902. I. Sheep Feeding on the Range. II. Lamb FeedingSecond Trial.

No. 52-April, 1902. Experiments in Evaporation. 

